\title{
Vertical transmission of group B Streptococcus and associated factors among pregnant women: a cross-sectional study, Eastern Ethiopia
}

This article was published in the following Dove Press journal: Infection and Drug Resistance

\author{
Tesfaye Assebe Yadeta' \\ Alemayehu Worku² \\ Gudina Egata ${ }^{3}$ \\ Berhanu Seyoum ${ }^{4}$ \\ Dadi Marami ${ }^{4}$ \\ Yemane Berhane ${ }^{5}$ \\ 'School of Nursing and Midwifery, \\ College of Health and Medical \\ Sciences, Haramaya University, \\ Harar, Ethiopia; ${ }^{2}$ Department of \\ Epidemiology and Biostatistics, \\ School of Public Health, Addis \\ Ababa University, Addis Ababa, \\ Ethiopia; ${ }^{3}$ School of Public Health, \\ College of Health and Medical \\ Sciences, Haramaya University, \\ Harar, Ethiopia; ${ }^{4}$ Department of \\ Medical Laboratory Science, College \\ of Health and Medical Sciences, \\ Haramaya University, Harar, Ethiopia; \\ ${ }^{5}$ Department of Epidemiology, Addis \\ Continental Institute of Public Health, \\ Addis Ababa, Ethiopia
}

Background: Vertically transmitted group B Streptococcus (GBS) causes fetal and neonatal infections. However, there is limited information on the vertical transmission of GBS in lowincome countries. This study, therefore, aimed to determine the rate of vertical transmission of GBS and associated factors among pregnant women in Eastern Ethiopia.

Subjects and methods: A cross-sectional, facility-based study was conducted among pregnant women in Harar town, Eastern Ethiopia, from June to October, 2016. GBS positivity of pregnant women was confirmed by culture of rectovaginal swab. Vertical transmission at birth was confirmed by culture on swabs taken from the ear canal, umbilicus, axilla, groin, and nose within 6 hours after birth. Prevalence ratio (PR) along with 95\% CI was estimated to examine factors associated with vertical transmission using $\log$ binomial regression analysis.

Results: Out of 231 GBS-colonized pregnant women at delivery, 104 births were identified as GBS colonized with a vertical transmission rate of $45.02 \%$ and $95 \%$ CI: 38.49, 51.68. Of 104 vertical transmission cases, $65(62.50 \%)$ received no intrapartum antibiotic prophylaxis (IAP), $28(26.92 \%)$ received it $<4$ hours before delivery, and $11(10.58 \%)$ received it $\geq 4$ hours before delivery. Pre-labor rupture of membranes at term (PR: 1.93; 95\% CI: 1.04, 3.57), prolonged rupture of the membrane $\geq 18$ hours (PR: $1.76 ; 95 \% \mathrm{CI}: 1.13,2.74$ ), intrapartum maternal fever (PR: 1.40; 95\% CI: 1.13, 1.75), and IAP received $\geq 4$ hours (PR: 0.17; 95\% CI: 0.09, 0.30) were significantly associated with vertical transmission of GBS.

Conclusion: The magnitude of vertical transmission of GBS was very high. However, the rate of adequate IAP received by mothers was very low. Efforts need to be strengthened to screen pregnant women during antenatal care and IAP should be used as necessary. Furthermore, maternal vaccination may provide a feasible strategy to reduce the vertical transmission.

Keywords: GBS, vertical transmission, newborn, log binomial, Harar, at birth, IAP

\section{Introduction}

Group B Streptococcus (GBS) is a gram-positive bacterium. ${ }^{1}$ It causes invasive newborn and fetal infection. Early-onset neonatal disease (EOND) occurs in the first week of life (0-6 days), mostly associated with sepsis. ${ }^{2}$ Vertical transmission of GBS can occur during pregnancy or the birth process from genitourinary or gastrointestinal tract of colonized pregnant women. ${ }^{3}$ GBS colonizing the rectum and vagina of pregnant women ascend and cause chorioamnionitis, leading to fetal and neonatal colonization. ${ }^{4}$ Mortality risk among newborns with GBS colonization was 6.6-fold higher than for those without GBS. ${ }^{5}$ EOND occurs in 1.4 per 1,000 neonates in the general population and 7.8 per 1,000 among GBS-colonized women. ${ }^{6}$
Correspondence: Tesfaye Assebe Yadeta School of Nurse and Midwifery, College of Health and Medical Sciences, Haramaya University, PO Box 235, Harar, Ethiopia Tel +251911705707

Fax +25125666808 I

Email tesfaye.assebe@yahoo.com 
Neonates with early onset of GBS disease are more likely to have respiratory distress disease and convulsions and require longer hospital stays. ${ }^{7}$ Case fatality is highest in EOND when it occurs within 24 hours of birth. ${ }^{4}$ Survivors suffer from uncontrolled seizures, impaired psychomotor development, profound mental retardation, blindness, and deafness. ${ }^{8}$

Worldwide, vertical transmission of GBS shows considerable variation within and between geographic regions, ranging from $11.2 \%$ to $57.7 \%{ }^{9,10}$ The identified risk factors for vertical transmission were maternal fever during labor, prolonged rupture of the membrane (PROM), preterm delivery, chorioamnionitis, maternal GBS sepsis, and intrapartum antibiotic prophylaxis (IAP). ${ }^{11,12}$

IAP for women colonized with GBS can significantly decrease vertical transmission. ${ }^{13}$ IAP can also significantly reduce the rates of EOD and case fatality rate. ${ }^{6,14}$ The effectiveness of IAP was high if IAP was administered at least 4 hours before delivery. Early recognition and prompt treatment are crucial in preventing rapid invasive GBS disease progression. ${ }^{15}$

Countries that introduced prevention strategies including universal routine antenatal GBS screening, identification of risk factors, and offering antibiotic prophylaxis at delivery were able to significantly reduce the disease. ${ }^{16,17}$ However, most of the sub-Saharan African countries do not have clear guidelines for prevention, despite the high rate of vertical transmission. ${ }^{10,18,19}$ This could be due to lack of information on the vertical transmission of GBS. Thus, this study aimed to investigate the magnitude of vertical transmission of GBS among colonized women at birth and factors that are associated with vertical transmission.

\section{Subjects and methods Study setting}

The study was conducted in three selected health facilities in Harar town, Eastern Ethiopia. Harar town is located in Harari Regional State, which is one of the nine regional states of Ethiopia. The town is located $510 \mathrm{~km}$ away from Addis Ababa, the capital city of Ethiopia. The total population of the town is about 101,934 . In the town, there are six hospitals (four government and two private hospitals) and four health centers providing delivery services. For this study, Hiwot Fana Specialized Haramaya University Hospital, Jugal Hospital, and Arategna Health Centers were selected because they had the largest client load in their delivery services. ${ }^{20}$

\section{Study design and period}

Health facility-based cross-sectional study was conducted between June and October 2016. The study participants were pregnant women admitted to labor and delivery room at the selected health facilities for delivery. A total of 1,688 pregnant women in active labor were examined for rectovaginal GBS colonization. In this study, sample size was determined with the assumption of $95 \%$ CI to be used, power of the study as $80 \%$, control to case ratio of $2: 1$, percent of control (newborn not colonized) exposed (fever) as $2 \%$, and percent of case (newborn colonized) exposed as $26 \% .{ }^{11}$ Accordingly, a sample size of 81 mother-newborn pairs was calculated. However, during assessment of GBS colonization, which was designed for a different study, the total number of colonized pregnant women was found to be 231 (127 non-colonized newborn and 104 colonized newborn). To address other exposure variables and increase the power of the study, we included all the colonized mothers who were identified beyond the minimum requirement in the final analysis. Women who took antibiotics in the last 2 weeks during their pregnancy prior to specimen collection, pregnant women with antepartum hemorrhage, those women who were physically or mentally incapable of responding, and women with multiple pregnancies were excluded from the study. ${ }^{9}$

\section{Data collection}

Data were collected through interviewer-administered questionnaire, checklist for medical record, and laboratory test results. The questionnaire and checklist were adapted from the Ethiopian Demographic and Health Survey and the World Health Organization indicators of health status of mother during labor, delivery, and of immediate newborn. ${ }^{21,22}$ The questionnaire was initially prepared in English and then translated into the local languages Afaan Oromo and Amharic by language experts, and back into English to check its consistency.

Six days of training was given to data collectors, and their supervisors. For laboratory work, two medical microbiologists (to read, confirm, and interpret the test results) and three medical laboratory technologists (who assisted in media preparation and sterilization processes) were involved. A research manual was prepared for use during training, data collection, and data management.

Vertical transmission was the dependent variable in this study and understood as follows: it was confirmed when GBS was identified from both live born and stillbirths by swab culture from the ear canal, umbilicus, axilla, groin, or nose without signs or symptoms of infection within 6 hours of delivery from colonized women. ${ }^{4,9}$ Positive GBS was labeled as "yes" and negative GBS as "no" with a code of 1 and 0, respectively. Age, educational status, fever in 
current pregnancy, rupture of the membrane, gestational age, stillbirth, and intrapartum antibiotic administration were independent variables in this study.

Age of the mother was recorded based on maternal response later and was grouped as $15-25$ and 26-46 with codes 0 and 1, respectively, for analysis. Maternal education was grouped as "illiterate" for those who could not read and write and "literate" for all others, and was coded as 1 and 0 , respectively.

Data on rupture of membranes were obtained from medical records. PROM that took $\geq 18$ hours for delivery of the fetus, preterm premature rupture of membranes, and pre-labor rupture of membranes at term (term PROM) were labeled as "yes" and "no" later for analysis with codes 1 and 0 , respectively. For the purpose of this study, a temperature of $\geq 37.5^{\circ} \mathrm{C}$ was considered as maternal fever at admission and was obtained from the medical records during labor, and was labeled as "yes" and "no" with codes 1 and 0, respectively. Data on stillbirths were obtained from medical records. Stillbirth was labeled as "yes" and "no" and coded with 1 and 0 , respectively.

The current vertical transmission prevention guidelines recommend administration of IAP 4 or more hours before delivery. According to the revised Centers for Disease Control and Prevention guidelines for the prevention of neonatal GBS disease, all pregnant women should be screened at $35 \pm 37$ weeks of gestation for vaginal and rectal GBS colonization. IAP administration, at the time of labor or rupture of membranes, should be performed in several cases as follows: known maternal GBS colonization, preterm labor, preterm premature rupture of membranes, PROM exceeding 18 hours, infant with prior GBS disease, maternal intrapartum fever $\left(\geq 38^{\circ} \mathrm{C}\right)$, and women positive for GBS isolated from the urine at any time. ${ }^{23}$

Penicillin $\mathrm{G}$ is the preferred and recommended IAP agent that is given at $5 \mathrm{MU}$ intravenously (IV), then 2.5 MU IV every 4 hours; ampicillin administered at $2 \mathrm{~g} I \mathrm{~V}$, then $1 \mathrm{~g}$ every 4 hours until delivery is the other choice. Beta-lactam allergic patients receive erythromycin or clindamycin IV in an equivalent dosage. Women with reported beta-lactam allergy, but at low risk for anaphylaxis, should receive cefazolin $2 \mathrm{~g} \mathrm{IV}$, then $1 \mathrm{~g}$ every 8 hours, while those at high risk of anaphylaxis (prior history of anaphylaxis, angioedema, respiratory distress, or urticaria following administration of a penicillin or cephalosporin) should receive clindamycin $900 \mathrm{mg}$ IV every 8 hours (if the GBS is susceptible) or vancomycin $1 \mathrm{~g}$ IV every 12 hours. ${ }^{23}$

Pregnant women who received IAP $<4$ hours before delivery were grouped as inadequate. ${ }^{15}$ Data for IAP administration were also taken from the medical record and labeled as "yes" and "no" and coded with 1 and 0, respectively. Duration of IAP from the first time of administration to delivery was taken from the medical record and then estimated for $\geq 4$ and $<4$ hours. Later, for the regression analysis, women who had not received and received inadequate IAP were grouped as "no and inadequate" and recorded as " 0 " and those who received adequate IAP were recorded as " 1 ".

For specimen collection, transportation, and processing, we followed the Centers for Disease Control and Prevention recommendations and the Standard Operating Procedures previously described elsewhere. ${ }^{23,24}$ In brief, within 6 hours after delivery, swab specimens were collected by the trained midwifery nurse at least from two sites (the ear canal, umbilicus, axilla, groin, or nose) of the stillborn and alive neonate using sterile cotton applicators. Soon after collection, the specimens were placed in sterile screw-capped test tubes containing Amie's transporting media with charcoal (Copan Diagnostics, Inc., New York, NY, USA) to sustain the viability of bacteria and transported to the Microbiology Laboratory of the Department of Medical Laboratory Sciences, College of Health and Medical Sciences, Haramaya University within 4 hours of collection in cold chain $\left(+4^{\circ} \mathrm{C}\right)$. Subsequently, the specimens were inoculated in Todd-Hewitt broth (Oxoid, Hampshire, UK), an enrichment broth for GBS, supplemented with gentamycin $(8 \mathrm{mg} / \mathrm{mL})$ and nalidixic acid (15 mg/mL; Biomerieux, France) to prevent the overgrowth of commensals, normal flora, and contaminants. The broth cultures were incubated aerobically at $35^{\circ} \mathrm{C}-37^{\circ} \mathrm{C}$. After overnight incubation, the specimen was aseptically subcultured onto 5\% sheep blood agar plates (Oxoid) and incubated at $35^{\circ} \mathrm{C}-37^{\circ} \mathrm{C}$ in an anaerobic condition. The cultures were examined by microbiologists on the following day, and all negative plates were reincubated additionally for 18-24 hours and inspected again. Suspected isolates of GBS were identified by a combination of colony morphology, gram staining, and biochemical tests such as sodium hippurate hydrolysis (Oxoid), catalase test (Oxoid), and Christie-Atkins-MunchPetersen (Oxoid) reaction test. Finally, all $\beta$-hemolytic, Gram-positive cocci, hippurate hydrolysis-positive, catalasenegative, and Christie-Atkins-Munch-Petersen test-positive isolates were reported as GBS.

\section{Statistical analysis}

The data were double entered and cleaned using Epi-Data Version 3.1 and analyzed using STATA version 13. Log binomial regression model was used to assess the association between independent variables and the outcome variable 
(vertical transmission). Log binomial regression directly estimates the prevalence ratio (PR) in binary and common outcome variables. The log binomial estimate is found to be the "best" estimate since it maximizes the correct binomial likelihood. ${ }^{25}$ Possible interactions between covariates were tested. The explanatory variables were tested for multicollinearity before entering them into the multivariable model using the variance inflation factor test, the tolerance test, and values of the standard error. No multicollinearity problem was found. Correlation matrix and covariate matrix were tested for the final model. Log likelihood ratio test, and Akaike's information criterion and Bayesian information criterion were used to select the final model. The Hosmer-Lemeshow goodness-of-fit tests were used to test for model fitness. ${ }^{26}$

Crude PRs with 95\% CIs were estimated to assess the association between each independent variable and the outcome variable, and a $p$ value was determined. Variables with $p$ value $\leq 0.2$ in the bivariate analysis were considered in the multivariable analysis by assuming that the outcome varies according to risk factors, along with maternal age and wellknown predictors of vertical transmission, regardless of the cut-off point for $p$ value. Adjusted PR (APR) along with 95\% CIs were estimated to assess the strength of the association, and a $p$ value $<0.05$ was used to declare the level of statistical significance in the multivariable analysis.

\section{Ethical considerations}

The study was reviewed and approved by the Institutional Health Research Ethics Review Committee of the Colleges of Health and Medical Sciences, Haramaya University. Written consent was obtained from each woman before commencement of data collection. Illiterate mothers gave their consent by their thumbprint, which is approved by the ethics review committee. Participants under the age of 18 provided their assent in addition to written or thumbprint consent of their parent or legal guardian. To maintain confidentiality, interviews and specimen collection were conducted in a separate room and all information obtained in this study was handled with anonymity. Colonized women were provided antibiotic prophylaxis, and neonates with health problems were treated and immediate referral was also facilitated.

\section{Results}

A total of 1,688 pregnant women were screened for rectovaginal GBS colonization at labor and delivery room. Of these, 231 (13.68\%) pregnant women were culture positive for GBS. In this study, these 231 GBS-colonized pregnant women were used to identify vertical transmission and its associated factors. The mean $( \pm \mathrm{SD})$ age of women was 25.31 $( \pm 6.70)$ years. More than half of the mothers (128 [55.41\%]) had no formal education, 108 (46.75\%) of them were from rural areas, and in $50(21.65 \%)$, the parity was five and above. Proportion of vertical transmission at delivery was 104/231 (45.02\%; 95\% CI: 38.49, 51.68). The proportions of vertical transmission at birth from a total of stillbirths and live births were $31 / 59$ (52.54\%) and 73/172 (42.44\%), respectively. The mean $( \pm S D)$ gestational age and the mean $( \pm S D)$ birth weight of the newborn were 37.89 weeks $( \pm 2.21)$ and $3,022.14 \mathrm{~g}$ ( \pm 581.69$)$, respectively. Among 231 newborns, 30 (12.99\%) were born preterm ( $<37$ weeks of gestation). Of these 104 colonized births, $39(37.50 \%)$ had been exposed to IAP and $65(62.50 \%)$ had no exposure to IAP. Of the 39 IAP exposed, $28(71.80 \%)$ were exposed $<4$ hours and $11(28.20 \%)$ were exposed $\geq 4$ hours. Of all newborns, those exposed to IAP $\geq 4$ hours were not colonized. However, all newborns who were exposed to IAP $<4$ hours were colonized (Table 1).

\section{Predictors of vertical transmission}

In univariable regression analysis, the following variables were associated with vertical transmission: term PROM, PROM $\geq 18$ hours, birth from mothers who were febrile during labor and delivery, and birth from mothers who received adequate IAP (Table 2).

To estimate the relative contribution of each factor to vertical transmission of GBS, APR was derived from log binomial regression models. Term PROM (APR: 1.93; 95\% CI: 1.04, 3.57), PROM (APR: 1.76; 95\% CI: 1.13, 2.74), and intrapartum maternal fever (APR: 1.40; 95\% CI: 1.13 , $1.75)$ were significantly associated with vertical transmission. Adequate IAP received ( $\geq 4$ hours) by mothers compared to not receiving or receiving inadequate IAP ( $<4$ hours) decreases the PR of vertical transmission by $83 \%$ (APR: 0.17; 95\% CI: 0.09, 0.30), as shown in Table 2 .

\section{Discussion}

In this study, vertical transmission of GBS from colonized mothers at birth was $45.02 \%$. Factors that were significantly associated with vertical transmission were term PROM, PROM $\geq 18$ hours before delivery, mothers having fever during labor, and mothers having received adequate IAP.

The vertical transmission rate reported in this study was consistent with those reported in studies from other lowincome countries. ${ }^{4,10,18}$ However, it was clearly higher than those reported from high-income countries where a guideline for GBS prevention is commonly available. ${ }^{9,11}$ These discrepancies might also be due to variations in demographic 
Table I Characteristics of the study participants by vertical transmission at birth in Harar, Eastern Ethiopia, $2016(\mathrm{~N}=23 \mathrm{I})$

\begin{tabular}{|c|c|c|c|}
\hline \multirow[t]{2}{*}{ Characteristics } & \multicolumn{3}{|c|}{ GBS test results at delivery } \\
\hline & Total & $\begin{array}{l}\text { Negative, } \\
\text { n (\%) }\end{array}$ & $\begin{array}{l}\text { Positive, } \\
\text { n (\%) }\end{array}$ \\
\hline \multicolumn{4}{|c|}{ Age of mothers (in years) } \\
\hline $15-25$ & $124(53.68)$ & 71 (57.26) & $53(42.74)$ \\
\hline $26-46$ & $107(46.32)$ & $56(52.34)$ & $5 \mathrm{I}(47.66)$ \\
\hline \multicolumn{4}{|l|}{ Residence } \\
\hline Urban & $123(53.25)$ & $65(52.85)$ & $58(47.15)$ \\
\hline Rural & $108(46.75)$ & $62(57.4 I)$ & $46(42.59)$ \\
\hline \multicolumn{4}{|c|}{ Educational level of mothers } \\
\hline Illiterate & $128(55.4 \mid)$ & $73(57.03)$ & $55(42.97)$ \\
\hline Literate & $103(44.59)$ & $54(52.43)$ & $49(47.57)$ \\
\hline \multicolumn{4}{|l|}{ Parity } \\
\hline Primiparous & $78(33.77)$ & $43(55.13)$ & $35(44.87)$ \\
\hline $1-4$ & $103(44.59)$ & $54(52.43$ & $49(47.57)$ \\
\hline$\geq 5$ & $50(21.65)$ & $30(60.00)$ & $20(40.00)$ \\
\hline \multicolumn{4}{|c|}{ Term pre-labor rupture of the membrane } \\
\hline No & $64(27.71)$ & $53(82.81)$ & II (I7.9I) \\
\hline Yes & $167(72.29)$ & $74(44.58)$ & $93(55.69)$ \\
\hline \multicolumn{4}{|c|}{ Rupture of membrane $\geq 18$ hours } \\
\hline No & $64(27.71)$ & $50(78.13)$ & $14(2 \mid .88)$ \\
\hline Yes & $167(72.29)$ & $77(46.11)$ & $90(53.89)$ \\
\hline \multicolumn{4}{|c|}{ Fever during labor } \\
\hline No & $136(58.87)$ & $94(69.12)$ & $42(30.88)$ \\
\hline Yes & $95(4 \mid .13)$ & $33(34.74)$ & $62(65.26)$ \\
\hline \multicolumn{4}{|c|}{ Preterm pre-labor rupture of the membrane } \\
\hline No & $207(89.61)$ & $116(56.04)$ & 91 (43.96) \\
\hline Yes & $24(10.39)$ & II (45.83) & $13(54.17)$ \\
\hline \multicolumn{4}{|c|}{ Gestational age, weeks } \\
\hline$\geq 37$ & $20 I(87.01)$ & $112(55.72)$ & $89(44.28)$ \\
\hline$<37$ & $30(12.99)$ & $15(50.00)$ & $15(50.00)$ \\
\hline \multicolumn{4}{|c|}{ APGAR score at fifth minute } \\
\hline$\geq 7$ & $136(57.14)$ & $78(57.35)$ & $58(42.65)$ \\
\hline$<7$ & $95(42.86)$ & $49(51.58)$ & $49(51.58)$ \\
\hline \multicolumn{4}{|l|}{ IAP } \\
\hline Yes & $|3|(56.7 \mid)$ & $92(70.23)$ & $39(29.77)$ \\
\hline No & $100(43.29)$ & $35(35.00)$ & $65(65.00)$ \\
\hline \multicolumn{4}{|c|}{ Time at which IAP before delivery, hours } \\
\hline$<4$ & $28(100.00)$ & 0 & $28(21.37)$ \\
\hline$\geq 4$ & $103(88.63)$ & $92(89.32)$ & II (I0.68) \\
\hline \multicolumn{4}{|c|}{ Women received IAP } \\
\hline No/inadequate & $128(100.00)$ & $35(27.34)$ & $93(72.66)$ \\
\hline Adequate & $103(88.63)$ & $92(89.32)$ & $\mathrm{II}(10.68)$ \\
\hline \multicolumn{4}{|l|}{ Stillbirth } \\
\hline No & $172(74.46)$ & $99(57.56)$ & $73(42.44)$ \\
\hline Yes & $59(25.54)$ & $28(47.46)$ & $31(52.54)$ \\
\hline \multicolumn{4}{|c|}{ Neonatal death within 6 hours } \\
\hline No & $225(97.40)$ & $124(55.11)$ & $101(44.89)$ \\
\hline Yes & $6(2.60)$ & $3(50.00)$ & $3(50.00)$ \\
\hline
\end{tabular}

Abbreviations: GBS, group B Streptococcus; IAP, intrapartum antibiotic prophylaxis.

characteristics, geographic location, and service availability. ${ }^{4}$ High rate of vertical transmission contributes to high neonatal and maternal morbidity and mortality due to GBS. Vertical transmission of GBS is preventable; health care providers and policymakers need to consider this in their maternal and neonatal mortality reduction strategies. ${ }^{5}$

Term PROM was also reported to have increased the risk of vertical transmission. ${ }^{12}$ GBS infection of the choriodecidua induces dysfunction of the cytokeratin network in amniotic epithelium, resulting in membrane weakening which causes early rupture of the membrane. ${ }^{27}$ Rupture of the membrane leaves the fetus and the amniotic fluid, which is a good medium for bacterial proliferation, unprotected and thus increases the risk of infection.

PROM ( $\geq 18$ hours) was also reported to have increased the risk of vertical transmission in GBS-colonized women. ${ }^{28}$ Prolonged exposure of fetus to maternal bacterial flora without any protective membrane barrier enhances the transmission, which can result in colonization of the fetus in the uterus in the absence of timely preventive measures. ${ }^{29}$

Women with both microbial invasion of the amniotic cavity and acute histologic chorioamnionitis had the highest intra-amniotic inflammatory response, which was mediated by interleukin- 6 concentrations. Fever is one of inflammatory response manifestations and increases the risk of neonatal colonization. ${ }^{11,12,30}$ During the delivery process, once the fetus is colonized by GBS, it is highly likely to develop pneumonia and/or sepsis. ${ }^{31}$ GBS can be isolated from cord blood and lung aspirates, and the GBS bacterial pigment/lipid toxin causes hemolysis or pyroptosis that exacerbates fetal damage and increases the risk of fetal loss. ${ }^{32}$

In this study, we found that mothers who received adequate IAP were significantly associated with decreased risk of vertical transmission, which is consistent with the findings of studies conducted elsewhere. ${ }^{33}$ IAP helps to prevent vertical transmission of GBS by reducing the bacterial load of rectum and vagina and, thus, preventing GBS from ascending and proliferating in the fetus and amniotic fluid compartment. ${ }^{34}$ In countries where there is low utilization of maternal care and in countries with resource-poor settings, feasibility of adequate IAP is challenging. ${ }^{19}$ Antisepsis at delivery has been revealed to be ineffective. ${ }^{35}$ However, maternal vaccination may provide a feasible strategy to reduce GBS disease in countries where there is low utilization of maternal care and in countries with resource-poor settings. ${ }^{36}$

In this study, all newborns exposed to IAP $\geq 4$ hours were found to be not colonized. However, all newborns exposed to IAP $<4$ hours were colonized. Sufficient antibiotic levels in the fetal bloodstream are required to prevent fetal and neonatal infection during labor. Four hours of IAP was needed to reduce the number of women with positive GBS cultures. ${ }^{15}$ GBS vaginal colony counts decreased by 5 -fold 
Table 2 Results of bivariate and multivariate log binomial model on factors associated with vertical transmission of GBS at birth in Harar, Eastern Ethiopia, 2016

\begin{tabular}{|c|c|c|c|c|c|}
\hline \multirow[t]{2}{*}{ Characteristics } & \multirow[t]{2}{*}{ Total, n (\%) } & \multicolumn{2}{|c|}{ Crude PR } & \multicolumn{2}{|c|}{ Adjusted PR } \\
\hline & & $\overline{\mathbf{P R}}$ & $(95 \% \mathrm{Cl})$ & PR & $(95 \% \mathrm{Cl})$ \\
\hline \multicolumn{6}{|c|}{ Age of mothers in years } \\
\hline $15-25$ & $124(53.68)$ & I & I & & \\
\hline $26-49$ & $107(46.32)$ & 0.89 & $0.67,1.19$ & 0.99 & $0.81,1.12$ \\
\hline \multicolumn{6}{|c|}{ Term pre-labor rupture of the membrane } \\
\hline No & $64(27.71)$ & 1 & & 1 & \\
\hline Yes & $167(72.29)$ & 3.24 & $1.86,5.64 *$ & 1.93 & $1.04,3.57^{*}$ \\
\hline \multicolumn{6}{|c|}{ Rupture of membrane $\geq 18$ hours } \\
\hline No & $64(27.71)$ & I & & I & \\
\hline Yes & 167(72.29) & 2.46 & I.5I, 3.99* & 1.76 & $1.13,2.74 *$ \\
\hline \multicolumn{6}{|c|}{ Fever during labor } \\
\hline No & $136(58.87)$ & I & & I & \\
\hline Yes & $95(4 \mid .13)$ & 2.11 & I.57, $2.82 *$ & 1.40 & $1.13,1.75 *$ \\
\hline \multicolumn{6}{|l|}{ Stillbirth } \\
\hline No & $172(74.46)$ & I & & 1 & \\
\hline Yes & $59(25.54)$ & 1.23 & $0.91,1.66$ & 1.12 & $0.86,1.38$ \\
\hline \multicolumn{6}{|c|}{ Women received IAP } \\
\hline No/inadequate & $128(55.4 \mid)$ & I & & I & \\
\hline Adequate & $103(44.59)$ & 0.14 & $0.08,0.25^{*}$ & 0.17 & $0.09,0.30^{*}$ \\
\hline \multicolumn{6}{|c|}{ Preterm pre-labor rupture of the membrane } \\
\hline No & $207(89.61)$ & I & & & \\
\hline Yes & $24(10.39)$ & 1.23 & $0.82,1.83$ & & \\
\hline \multicolumn{6}{|c|}{ Gestational age, weeks } \\
\hline$\geq 37$ & $20 \mathrm{I}(87.0 \mathrm{I})$ & I & & & \\
\hline$<37$ & $30(12.99)$ & 1.12 & $0.76,1.66$ & & \\
\hline \multicolumn{6}{|c|}{ Educational level of mothers } \\
\hline Illiterate & $128(55.4 \mid)$ & I & & & \\
\hline Literate & $103(44.59)$ & 0.9 & $0.67,1.20$ & & \\
\hline \multicolumn{6}{|c|}{ APGAR score at fifth minute } \\
\hline$\geq 7$ & $136(57.14)$ & I & & & \\
\hline$<7$ & $95(42.86)$ & 1.13 & $0.85,1.50$ & & \\
\hline
\end{tabular}

Note: ${ }^{*} p \leq 0.05$.

Abbreviations: GBS, group B Streptococcus; IAP, intrapartum antibiotic prophylaxis; PR, prevalence ratio.

within 2 hours of administering IV penicillin G and by 50 -fold within 4 hours. ${ }^{34}$ Delay in administering IAP due to factors such as late presentation to the labor ward, precipitous delivery, or misidentification of risk factors can result in inadequate IAP cover. ${ }^{19}$

In Ethiopia, $34.3 \%$ of neonatal mortality is due to infections. ${ }^{37}$ The proportion of GBS-related neonatal death has not been investigated. High proportion (72\%) of women give birth at home. ${ }^{21}$ Maternal knowledge on the risk factors is low. ${ }^{38}$ Furthermore, there are no clear guidelines for prevention of specific GBS vertical transmission. The results of this study throw light on the current initiatives that are focused on improving maternal and newborn health care delivery system, the magnitude of the problem, and call for the necessary actions to be taken in order to strengthen efforts to reduce neonatal mortality.
To our knowledge, this is the first study to present vertical transmission of GBS. We used a questionnaire that was adapted from the World Health Organization and the Ethiopian Demographic and Health Survey pregnancy and pregnancy outcome indicators. Data collectors were thoroughly trained. Specimen collection and interviews were conducted in private places to ensure privacy. Although some studies collected swabs from only the vagina to identify GBS-colonized mothers, we have collected from both rectum and vagina, which increases the detection rate. ${ }^{11,39}$ Moreover, some studies collected swabs from a single anatomic site of newborn to identify vertical transmission, whereas we collected from the ear canal, umbilical area, axilla, groin, or nose to identify vertical transmission. ${ }^{9}$ To preserve better viability and stability of GBS, specimens were transported to the laboratory, inoculated into broth media, and incubated within 4 hours of specimen collection. The log 
binomial that estimates PR in cross-sectional studies and a common ( $>10 \%$ ) binary outcome variable is more appropriate than logistic regression that estimates odds ratio, as odds ratio commonly overestimates PR in binary outcome variables. However, ORs were more frequently reported than PRs. ${ }^{11}$

This study was not without limitations. Interviewer and recorder bias might have occurred during the interviews and recording. However, supervisor and principal investigators rechecked for the completeness of data before the mother and newborn were discharged and their card was returned back to the main record office. Measurement and diagnostic bias might also have occurred during measurement and diagnoses; however, two professionals were involved in measuring and reading the results. Moreover, in case of disagreement, investigators were also involved in the decision. Serotyping and molecular tests were not done for identified strains from mother-newborn pairs to further characterize the accordance (same or similar) of GBS by serotype and gene. As the study was done in health facilities, the findings may not able to be generalized to the entire community.

In conclusion, in this study, the prevalence of vertical transmission of GBS was found to be high. However, the number of pregnant women who received adequate IAP was very low. Initiating and strengthening GBS screening and introducing timely IAP are necessary. Furthermore, maternal vaccination may provide a feasible strategy to reduce vertical transmission.

\section{Acknowledgments}

The authors would like to thank Haramaya University for funding this study. They would also like to thank the study participants, data collectors, and staff of the Department of Medical Laboratory Sciences, College of Health and Medical Sciences for their kind cooperation.

\section{Author contributions}

All authors contributed toward data analysis, drafting and revising the paper and agree to be accountable for all aspects of the work.

\section{Disclosure}

The authors report no conflicts of interest in this work.

\section{References}

1. McGee L, Beall B. Streptococci. In: de Filippis I, McKee ML, editors. Molecular typing in bacterial infections. New York: Springer; 2013:109-126.

2. Bekker V, Bijlsma MW, van de Beek D, Kuijpers TW, van der Ende A. Incidence of invasive group B streptococcal disease and pathogen genotype distribution in newborn babies in the Netherlands over 25 years: a nationwide surveillance study. Lancet Infect Dis. 2014;14(11):1083-1089.
3. Scasso S, Laufer J, Rodriguez G, Alonso JG, Sosa CG. Vaginal group B streptococcus status during intrapartum antibiotic prophylaxis. Int $J$ Gynecol Obstet. 2015;129(1):9-12.

4. Seale AC, Koech AC, Sheppard AE, et al. Maternal colonization with Streptococcus agalactiae and associated stillbirth and neonatal disease in coastal Kenya. Nat Microbiol. 2016;1(7):16067.

5. Islam MS, Saha SK, Islam M, et al. Prevalence, serotype distribution and mortality risk associated with group B Streptococcus colonization of newborns in rural Bangladesh. Pediatr Infect Dis J. 2016;35(12):1309-1312.

6. Petersen KB, Johansen HK, Rosthoj S, et al. Increasing prevalence of group B streptococcal infection among pregnant women. Dan Med J. 2014;61(9):A4908.

7. Al-Kadri HM, Bamuhair SS, Al Johani SM, Al-Buriki NA, Tamim HM Maternal and neonatal risk factors for early-onset group B streptococcal disease: a case control study. Int $J$ Womens Health. 2013;5:729-735.

8. Dangor Z, Lala SG, Cutland CL, et al. Burden of invasive group B Streptococcus disease and early neurological sequelae in South African infants. PLoS One. 2015;10(4):e0123014.

9. Kunze M, Ziegler A, Fluegge K, Hentschel R, Proempeler H, Berner R. Colonization, serotypes and transmission rates of group B streptococci in pregnant women and their infants born at a single University Center in Germany. J Perinatal Med. 2011;39(4):417-422.

10. Le Doare K, Jarju S, Darboe S, et al. Risk factors for Group B Streptococcus colonisation and disease in Gambian women and their infants. J Infect. 2016;72(3):283-294.

11. Berardi A, Rossi C, Guidotti I, et al. Factors associated with intrapartum transmission of group B streptococcus. Pediatr Infect Dis $J$. 2014;33(12):1211-1215.

12. Shah D, Saxena S, Randhawa VS, Nangia S, Dutta R. Prospective analysis of risk factors associated with group B streptococcal colonisation in neonates born at a tertiary care centre in India. Paediatri Int Child Health. 2014;34(3):184-188.

13. Berardi A, Rossi C, Creti R, et al. Group B streptococcal colonization in 160 mother-baby pairs: a prospective cohort study. $J$ Pediatr. 2013;163(4):1099-1104. e1091.

14. Fairlie T, Zell ER, Schrag S. Effectiveness of intrapartum antibiotic prophylaxis for prevention of early-onset group B streptococcal disease. Obstet Gynecol. 2013;121(3):570-577.

15. Kojima K, Tanaka R, Nakajima K, et al. Predicting outcomes of neonates born to GBS-positive women who received inadequate intrapartum antimicrobial prophylaxis. Turk J Pediatr. 2014;56(3):238-242.

16. Homer CS, Scarf V, Catling C, Davis D. Culture-based versus risk-based screening for the prevention of group B streptococcal disease in newborns: a review of national guidelines. Women Birth. 2014;27(1):46-51.

17. Darlow B, Campbell N, Austin N, et al. The prevention of early-onset neonatal group B streptococcus infection: New Zealand Consensus Guidelines 2014. N Z Med J. 2015;128(1425):69-76.

18. Madzivhandila M, Adrian PV, Cutland CL, Kuwanda L, Schrag SJ, Madhi SA. Serotype distribution and invasive potential of group B streptococcus isolates causing disease in infants and colonizing maternal-newborn dyads. PLoS One. 2011;6(3):e17861.

19. Nishihara Y, Dangor Z, French N, Madhi S, Heyderman R. Challenges in reducing group B Streptococcus disease in African settings. Arch Dis Child. 2016;102(1):72-77.

20. Federal Democratic Republic of Ethiopia Population Census Commission. Summary and Statistical Report of the 2007 Population and Housing Census Results. Addis Ababa, Ethiopia: Central Statistical Authority (CSA); 2008.

21. Central Statistical Agency (CSA) [Ethiopia] and ICF. Ethioia Demographic and Health Survey: Key Indicator Report. Addis Ababa, Ethiopia, and Rockville, Maryland, USA: CSA and ICF; 2016.

22. Maine D. Monitoring emergency obstetric care: a handbook. Geneva, Switzerland World Health Organization; 2009.

23. Verani JR, McGee L, Schrag SJ. Prevention of perinatal group B streptococcal disease: revised guidelines from CDC, 2010. Recomm Rep. 2010;59(10):1-32.

24. Nahler G, Nahler G. Standard operating procedures (SOP). Wien Austria: Springer; 2009. 
25. Williamson T, Eliasziw M, Fick GH. Log-binomial models: exploring failed convergence. Emerg Themes Epidemiol. 2013;10(1):14.

26. Blizzard L, Hosmer W. Parameter estimation and goodness-of-fit in log binomial regression. Biom J. 2006;48(1):5-22.

27. Vanderhoeven JP, Bierle CJ, Kapur RP, et al. Group B streptococcal infection of the choriodecidua induces dysfunction of the cytokeratin network in amniotic epithelium: a pathway to membrane weakening. PLoS Pathog. 2014;10(3):e1003920.

28. Hamedi A, Akhlaghi F, Seyedi SJ, Kharazmi A. Evaluation of group B Streptococci colonization rate in pregnant women and their newborn. Acta Med Iran. 2012;50(12):805-808.

29. Edwards RK, Tang Y, Raglan GB, Szychowski JM, Schulkin J, Schrag SJ. Survey of American obstetricians regarding group B streptococcus: opinions and practice patterns. Am J Obstet Gynecol. 2015;213(2):229. e1-e7.

30. Kacerovsky M, Musilova I, Andrys C, et al. Prelabor rupture of membranes between 34 and 37 weeks: the intraamniotic inflammatory response and neonatal outcomes. Am J Obstet Gynecol. 2014;210(4):325. e321-325.

31. Lin FY, Troendle JF. Hypothesis: neonatal respiratory distress may be related to asymptomatic colonization with group B streptococci. Pediatr Infect Dis J. 2006;25(10):884-888.

32. Whidbey C, Vornhagen J, Gendrin C, et al. A streptococcal lipid toxin induces membrane permeabilization and pyroptosis leading to fetal injury. EMBO Mol Med. 2015;7(4):488-505.
33. Turrentine M. Intrapartum antibiotic prophylaxis for Group B Streptococcus: has the time come to wait more than 4 hours? Am J Obstet Gynecol. 2014;211(1):15-17.

34. McNanley AR, Glantz JC, Hardy DJ, Vicino D. The effect of intrapartum penicillin on vaginal group B streptococcus colony counts. Am JObstet Gynecol. 2007;197(6):583. e1-e4.

35. Ohlsson A, Shah VS, Stade BC. Vaginal chlorhexidine during labour to prevent early-onset neonatal group $\mathrm{B}$ streptococcal infection. Cochrane Database Syst Rev. 2014;12:CD003520.

36. Madhi SA, Dangor Z. Prospects for preventing infant invasive GBS disease through maternal vaccination. Vaccine. 2017;35(35 Pt A): 4457-4460.

37. Debelew GT, Afework MF, Yalew AW. Determinants and causes of neonatal mortality in Jimma zone, southwest Ethiopia: a multilevel analysis of prospective follow up study. PLoS One. 2014;9(9): e107184.

38. Yadeta TA, Kumsa FA. Awareness and health care practice of mothers' about obstetric danger signs at Haramaya District, Eastern Ethiopia. $J$ Fam Med Health Care. 2017;3(1):23-29.

39. Moussa TA, Elsherif RH, Mohamed YA, Dawoud ME, AboElAref AM. Group B streptococcus colonization of pregnant women: comparative molecular and microbiological diagnosis. Comp Clin Path. 2013;22(6):1229-1234
Infection and Drug Resistance

\section{Publish your work in this journal}

Infection and Drug Resistance is an international, peer-reviewed openaccess journal that focuses on the optimal treatment of infection (bacterial, fungal and viral) and the development and institution of preventive strategies to minimize the development and spread of resistance. The journal is specifically concerned with the epidemiology of antibiotic
Dovepress

resistance and the mechanisms of resistance development and diffusion in both hospitals and the community. The manuscript management system is completely online and includes a very quick and fair peerreview system, which is all easy to use. Visit http://www.dovepress.com/ testimonials.php to read real quotes from published authors. 